\title{
Effect of Annealing on Low-Temperature Thermal Conductivity of High-Density Polyethylene
}

\author{
Yasuaki KAWAMURA* \\ Department of Applied Physics, Faculty of Engineering, \\ University of Tokyo, Bunkyo-ku, Tokyo, Japan.
}

(Received May 22, 1970)

\begin{abstract}
Thermal conductivity of high-density polyethylene was measured by the steady-flow method at temperatures from 4.2 to $20^{\circ} \mathrm{K}$. Results were analysed assuming two scattering processes of phonons: grain boundary scattering with a mean free path $\Lambda_{G}$ which is independent of phonon wave number $k$ and intragrain scattering with a mean free path $A_{\mathrm{D}}=A / a k^{2}$. It was found that $\Lambda_{\mathrm{G}}=0.48 \mu$ and $A / a=0.51 \times 10^{10} \mathrm{~cm}^{-1}$ for the as-received sample, and both increase with annealing. The radius of spherulite which was kept constant with annealing ranged 5 to $10 \mu$. $\Lambda_{G}$ is attributed to the extension of lamellar crystallite in the $a b$-plane. $\Lambda_{\mathrm{D}}$ is assigned to intralamellar subboundaries. According to results in literature, the ratio of thermal conductivity $K$ to specific heat $C$ is almost constant from 1 to $3^{\circ} \mathrm{K}$, where the grain boundary scattering is predominant. Since at $1^{\circ} \mathrm{K}$ the contribution of two-dimensional lattice waves (Lamb waves in lamellae) is more appreciable than three-dimensional ones, the above result implies that $\Lambda_{G}$ is the same for the two types of waves, which is consistent with our conclusion for $\Lambda_{\mathrm{G}}$.
\end{abstract}

KEY WORDS Polyethylene/Thermal Conductivity/Annealing/Defect/ Grain Boundary $/ 4.2-20^{\circ} \mathrm{K} /$

Thermal conductivity of insulator crystals is limited by various scattering processes of lattice waves or phonons. At high temperatures the anharmonicity of lattice vibrations is responsible for the phonon-phonon interaction process (umklapp process). ${ }^{1}$ Since, at these temperatures, phonon density is proportional to absolute temperature $T$ and the scattering probability is inversely proportional to phonon density, the mean free path arising from the umklapp process is inversely proportional to $T$. Therefore, at high temperatures where specific heat is considered to be constant in accordance with DulongPetit's law, thermal conductivity increases with decreasing temperature in inverse proportion to $T$.

At lower temperatures, however, where the umklapp process is not appreciable, the effect of scattering processes of phonons by various structural defects in the material becomes predominant. Thus the conductivity has a maximum

* Present address: The Institute of Physical and Chemical Research, Yamatomachi, Saitama, Japan. at an intermediate temperature and then approaches zero when $T$ goes to absolute zero. Temperature dependence of the conductivity in the low-temperature range reflects the nature of defects. In the case of polycrystals, the defects include grain boundaries and other smaller defects in crystallites. Study of thermal conductivity at low-temperatures, therefore, will be useful for understanding the nature of defects.

Thermal conductivity of crystalline polymers at low temperatures was investigated for polyethylene, nylon, and polytetrafluoroethylene by Reese and Tucker, ${ }^{2}$ and for polyethylene and nylon by Kolouch and Brown. ${ }^{3}$ These authors analysed results by use of procedures by Chang and Jones, ${ }^{4}$ who assumed the conductivity to be limited by scattering of phonons by two kinds of defects, the grain boundaries and the two-dimensional defects in crystallites.

Reese and Tucker ${ }^{2}$ estimated the size of the defects from thermal conductivity but the entity of the defects is still open to question. The purpose of the present study is to clarify the 
nature of the defects from thermal conductivity in three kinds of high-density polyethylene prepared by succesive annealing.

\section{EXPERIMENTAL}

Measurement of thermal conductivity was carried out in the temperature range from 4.2 to $20^{\circ} \mathrm{K}$ by the steady flow method. ${ }^{3,5}$ Figure 1 shows the schematic diagram of the apparatus.

The sample was machined into a circular rod $10 \mathrm{~mm}$ in diameter and $100 \mathrm{~mm}$ in length. The top of the sample was screwed into a copper block and a small block of copper with a heater winding was attached to the bottom of the sample. The sample with the copper blocks was surrounded with an inner brass shield. The inner shield was further covered by an outer brass shield, to which the inner shield was fixed with stainless-steel pipes of high thermal resistance. Both shields were evacuated to $10^{-5}$

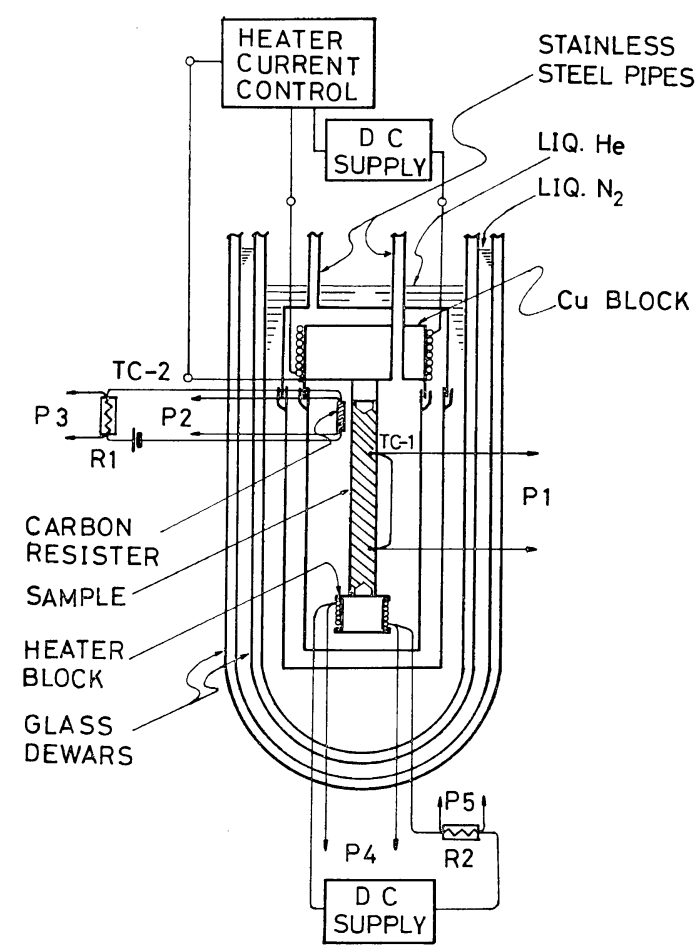

Figure 1. Schematic diagram of measuring system. P1 to P5 represent leads to the potentiometer, TC-1 and TC-2 thermocouples, R1 and R2 standard resistors.
mmHg to avoid heat transfer through conduction and convection. The outer shield was surrounded with liquid helium and the temperature was kept at $4.2^{\circ} \mathrm{K}$. Heat radiation from the sample was shielded by the inner jacket which was nearly the same temperature as the sample.

Temperature-control of the sample was carried out with a $200 \Omega$-heater of constantan wire wound on the upper copper block. The temperature of the copper block was detected with a Cu-constantan thermocouple (TC-2). The output from the TC-2 was fed to the control circuit $^{6,7}$ through which the heater current was controlled automatically.

A carbon resistor $(49 \Omega, 1 / 8 \mathrm{~W})$ was used for temperature measurement of the specimen. It was calibrated at three temperatures: ${ }^{7,8}$ liquid nitrogen temperature $\left(77^{\circ} \mathrm{K}\right)$; liquid helium temperature $\left(4.2^{\circ} \mathrm{K}\right)$, and $\lambda$-point of $\mathrm{He}$ which was obtained by lowering pressure.

A constant heat flux was supplied to the sample from the lower block by use of heater winding of $40 \Omega$, and the resultant temperature gradient along the sample was measured with a thermocouple of $\mathrm{Cu}-\mathrm{Au}$ : Co alloy (TC-1). The two junctions of TC-1 were embedded into the sample to the depth of $3 \mathrm{~mm}$ and with a separation of $60 \mathrm{~mm}$.

All the leads were thermally anchored to the upper copper block and drawn out through the steel pipes, by which the air inside the two shields was evacuated. These leads consisted of enamel-coated copper wire of $0.06 \mathrm{~mm} \phi$ except for a constantan wire of $0.2 \mathrm{~mm} \phi$ for the TC-2.

The thermal conductivity was calculated using the well known expression for the one-dimensional heat flow,

$$
\dot{Q}=K S\left(\frac{\Delta T}{\Delta x}\right)
$$

where $\dot{Q}$ is the heat flux into the sample, $K$ the thermal conductivity, $S$ the cross-sectional area of the sample, and $\Delta T / \Delta x$ the temperature gradient in the sample. The temperature difference between the two junctions was as small as about $2 \%$ of the temperature of the sample.

The measuring procedures were as follows. The two shields were first evacuated and then filled with helium gas at a pressure of $c a .200$ 


\section{Y. KaWAmURA}

Table I. Samples of polyethylene and results

\begin{tabular}{lccccc}
\hline \multicolumn{1}{c}{ Sample } & $\begin{array}{c}d \\
(\text { density at } \\
\left.30^{\circ} \mathrm{C}, \mathrm{g} / \mathrm{cm}^{3}\right)\end{array}$ & $\begin{array}{c}X_{c} \\
(\text { crystallinity, } \\
\%)\end{array}$ & $\begin{array}{c}u \\
\text { (sound velocity, } \\
\left.10^{5} \mathrm{~cm} / \mathrm{sec}\right)\end{array}$ & $\begin{array}{c}A / a, \\
A_{G}, \mu \\
10^{10} \mathrm{~cm}^{-1}\end{array}$ \\
\hline A (as-received) & 0.9642 & 75 & 2.15 & 0.48 & 0.51 \\
B (A annealed at $115^{\circ} \mathrm{C}$ for $\left.8 \mathrm{hr}\right)$ & 0.9662 & 80 & 2.20 & 1.4 & 0.71 \\
C (B annealed at $128^{\circ} \mathrm{C}$ for $\left.6 \mathrm{hr}\right)$ & 0.9686 & 82 & 2.23 & 2.0 & 0.75 \\
\hline
\end{tabular}

mmHg. After preliminary cooling with liquid nitrogen, liquid helium was introduced into the inner glass dewar and the system was left for two hours or less till thermal equilibrium was reached. Then the lower heater block was heated by a regulated dc power supply, and after the steady state was established, the temperature gradient in the sample was measured with a potentiometer. The temperature of the sample was then raised by heating the upper block, and after the new equilibrium was established, the next measurement was made. The interval between successive measurements was about two hours.

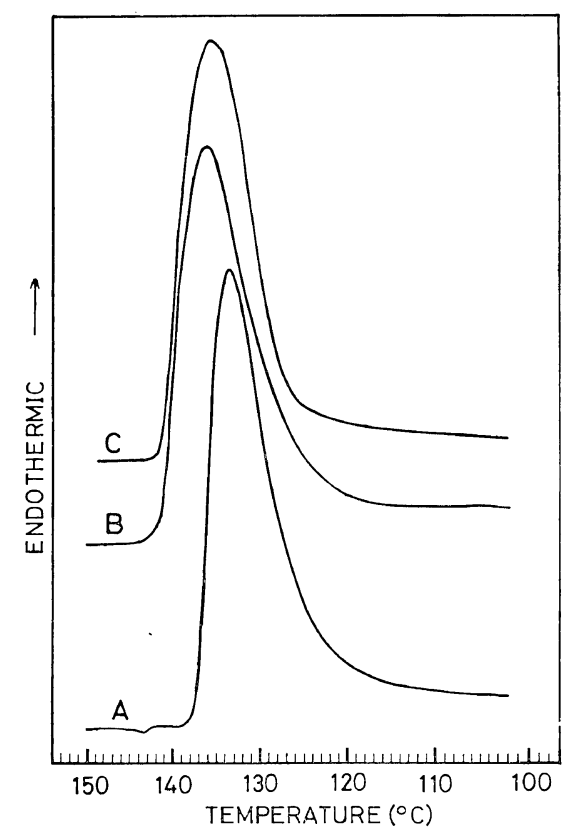

Figure 2. Heat flow plotted against temperature for three samples of polyethylene obtained by differential scanning calorimeter with scan speed $8 \mathrm{deg} / \mathrm{min}$. Symbols are the same as in Table I. Weight of sample, $14.1,13.8$, and $13.3 \mathrm{mg}$ for A to $\mathrm{C}$, respectively.
The sample used was high-density polyethylene (Hizex 2200). Conditions of heat-treatment of the sample are lisied in Table I. Effect of the heat-treatment was examined by thermal analysis with a differential scanning calorimeter. As is shown in Figure 2, the peak of the melting curve appears at about 133,136 and $136^{\circ} \mathrm{C}$ for samples $A, B$, and $C$, respectively. The shape of the peak becomes sharp in the same order. The density, which was measured with a densitygradient tube of benzene-carbon tetrachloride mixture at $30^{\circ} \mathrm{C}$, increased with annealing as shown in Table I. The diameter of spherulite, however, was found almost unchanged with annealing, ranging from 5 to 10 microns, as revealed by observation with the polarizing microscope.

\section{RESULTS AND DISCUSSION}

Figure 3 shows the thermal conductivity as a function of temperature for three samples. The thermal conductivity increases with increasing temperature for each sample, and when compared at a definite temperature, the conductivity increases with annealing.

The thermal conductivity $K$ of insulator crystals at low temperatures originates from lattice waves or phonons scattered by various defects. It follows therefore,

$$
K=\frac{1}{3} \int_{0}^{\infty} E(x) u \Lambda g \mathrm{~d} \omega
$$

where $E$ is the Einstein function with the argument $x=\hbar \omega / k_{\mathrm{B}} T, g$ the frequency distribution function of phonons, $A$ the mean free path of phonons, and $u$ the velocity of phonons or the sound velocity. The scattering processes at low temperatures are assumed, following Chang and Jones, ${ }^{4}$ to consist of two mechanisms: one the grain-boundary scattering, $\Lambda_{G}$, and the other the scattering by intragrain two-dimensional defects, 


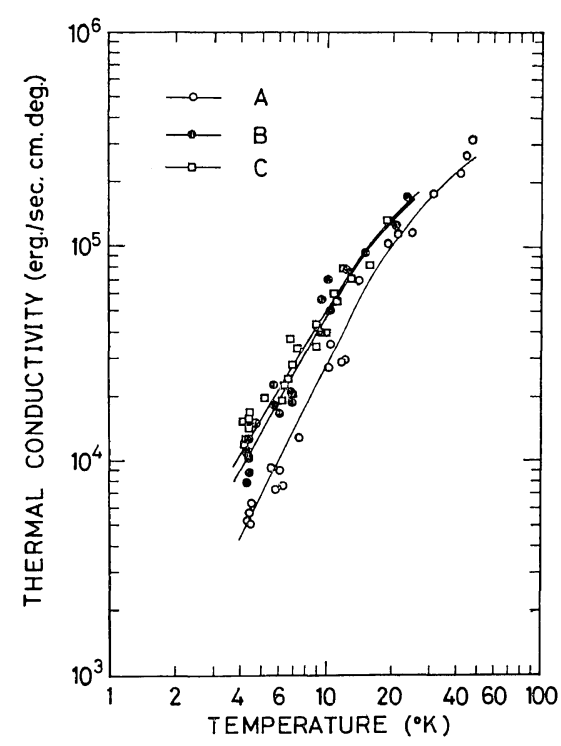

Figure 3. Thermal conductivity of polyethylene plotted against temperature. Symbols are the same as in Table I.

$\Lambda_{\mathrm{D}}$

$$
\frac{1}{\Lambda}=\frac{1}{\Lambda_{\mathrm{G}}}+\frac{1}{\Lambda_{\mathrm{D}}}
$$

$\Lambda_{\mathrm{G}}$ is assumed to be independent of $\omega$ and $T$ but $\Lambda_{\mathrm{D}}$ is a function of $\omega$ or phonon wavenumber $k$ as

$$
\Lambda_{\mathrm{D}}=\frac{A}{a k^{2}}
$$

The above expression is obtained by Klemens ${ }^{1,9}$ for two-dimensional defects of infinite extension lined parallel with a constant interval of $\gamma A a$, where $\gamma$ is a constant close to unity, $A a$ dimensionless constant and $a$ the lattice constant.

Substitution of eq 3 and 4 to 2 leads to

$$
\begin{aligned}
K= & \frac{k_{\mathrm{B}}{ }^{2} T}{6 \pi^{2} \hbar} \frac{A}{a} \int_{0}^{\infty} \frac{x^{4} e^{x}}{\left(e^{x}-1\right)^{2}} \\
& \times \frac{\mathrm{d} x}{x^{2}+\left(\frac{A}{a}\right)\left(\frac{1}{\Lambda_{G}}\right)\left(\frac{\hbar u}{k_{\mathrm{B}} T}\right)^{2}}
\end{aligned}
$$

in which, according to Debye, $g$ is assumed to be proportional to $\omega^{2}$. Since the temperature is much lower than the Debye temperature, the upper limit of the integral in eq 5 may be taken as infinity.
Two parameters in eq $5, \Lambda_{\mathrm{G}}$ and $A / a$, can be determined to obtain the best fit to the observed temperature dependence of $K$. The detailed process of curve fitting has been described by Chang and Jones. ${ }^{4}$ Sound velocity $u$ used in this process was calculated from low-temperature heat-capacity of polyethylene by Tucker and Reese,${ }^{10}$ assuming the Debye expression,

$$
C=\frac{2 \pi^{2} k_{\mathrm{B}}{ }^{4}}{5 \hbar^{3}}\left(\frac{T}{u}\right)^{3}
$$

Results are listed in Table I. The value of $\Lambda_{G}$ increases with annealing but is much smaller than the average diameter of spherulites which was kept constant with annealing. This indicates that the origin of $\Lambda_{G}$ is not the spherulite boundary.

The treatment of spherulitic polyethylene with fuming nitric acid indicates that lamellar crystallites have an extension of 1 micron or less in $a$ - and $b$-axes. ${ }^{11}$ Moreover, twisting of lamella often exists along the radial direction of a spherulite with a period of about 1 micron. ${ }^{12}$ These results suggest that the boundary of lamellae along $a$ - and $b$-axes may be the scatterer which determines $\Lambda_{\mathrm{G}}$. Since the sound velocity along $c$-axis is much higher than those along $a$ - and $b$-axes, and only low-frequency phonons are excited at low temperatures, the wavelength of phonons propagating along $c$-axis is much larger than the lamella thickness. The surface of lamellae may be, therefore, ineffective for scattering of phonons.

As can be seen in Table I, $A / a$ has the value of $10^{10} \mathrm{~cm}^{-1}$ in the order of magnitude, and increases with annealing. In the case of polyethylene, $a \simeq 3 \AA$ (the distance between nearest neighbor chains) and hence $A a \simeq 10^{3} \AA$ from the observed value of $A / a$. This would mean the defect responsible for $\Lambda_{\mathrm{D}}$ may be intra-lamellar sub-boundaries produced by the accumulation of dislocations. ${ }^{13-15}$ As is well known, as-grown polyethylene crystals include numerous dislocations. Screw dislocations run mostly in [110] planes $^{16}$ and edge dislocations parallel to $c$-axis, ${ }^{17}$ and as a result sub-boundaries form in the crystal. When the crystal is annealed, diffusion of molecules along chain direction occurs accompanying thickening of lamella. Dislocations will move in the course of annealing to mini- 


\section{Y. KAWAMURA}

mize the elastic energy and consequently the interval of sub-boundaries may increase.

\section{SPECIFIC HEAT AND THERMAL CONDUCTIVITY AT EXTREMELY LOW TEMPERATURES}

The specific heat of polyethylene at extremely low temperatures below $1^{\circ} \mathrm{K}$ shows the deviation from Debye's $T^{3}$-law. According to the data by Reese and Tucker, ${ }^{2}$ the deviation is enhanced with increasing crystallinity. The deviation results from the two-dimensional vibration of lamellar crystals which gives the specific heat proportional to $T^{2}$.

Recent study of polyethylene below $1^{\circ} \mathrm{K}$ by Giles and Terry ${ }^{18}$ revealed the thermal conductivity also deviates from the $T^{3}$-law and approaches the $T^{2}$-law with lowering temperature, indicating two-dimensional waves are effective to thermal conductivity.

Taking into account both three-dimensional and two-dimensional waves, it follows,

$K=\frac{1}{3} u_{3} \Lambda_{3} C_{3}+\frac{1}{3} u_{2} \Lambda_{2} C_{2}, \quad C=C_{3}+C_{2}, \quad$ ( 7 ) where $K$ is the thermal conductivity, $C$ the specific heat and suffices 3 and 2 represent threedimensional and two-dimensional waves, respectively. From the values ${ }^{2,10,18}$ for polyethylene of the same crystallinity, $K / C$ obtained is almost independent of temperature. $K / C$ was calculated as $0.5 \mathrm{~cm}^{2} / \mathrm{sec}$ at $1^{\circ} \mathrm{K}$ where $C_{2}$ is comparable with $C_{3}$ and $0.4 \mathrm{~cm}^{2} / \mathrm{sec}$ at $3^{\circ} \mathrm{K}$ where $C_{2} \ll C_{3}$. This implies that $u_{2} \Lambda_{2}$ is roughly equal to $u_{3} \Lambda_{3}$. From the elastic modulus of polyethylene crystals, the velocity of two-dimensional vibration (Lamb waves) ${ }^{19}$ was calculated to be 0.8 times that of bulk longitudinal waves. Therefore, we can conclude $\Lambda_{2} \simeq \Lambda_{3}$.

Since the two-dimensional waves in lamellae may be scattered by the boundary of lamella extension, that is, $\Lambda_{2}=\Lambda_{G}$, the above conclusion is consistent with the conclusion $\Lambda_{3}=\Lambda_{\mathrm{G}}$ described in the last section.

Acknowledgments. The author would like to thank Mr. H. Enjoji of Mitsubishi Petrochemical
Co., Ltd. very much for taking photographs of polyethylene samples with the polarizing microscope. Thanks are also due to Professors Y. Wada and K. Okano for their many stimulating discussions and continued encouragement throughout this work.

\section{REFERENCES}

1. P. G. Klemens, "Solid State Physics", Vol. 7 Academic Press, New York, 1958, p 1.

2. W. Reese and J. E. Tucker, J. Chem. Phys., 43, 105 (1965).

3. R. J. Kolouch and R. G. Brown, J. Appl. Phys., 39, 3999 (1968).

4. G. K. Chang and R. E. Jones, Phys. Rev., 126, 2055 (1962).

5. A. C. Anderson, W. Reese, and J. C. Wheatley, Rev. Sci. Instr., 34, 1386 (1963).

6. T. M. Dauphinee and S. B. Woods, Rev. Sci. Instr., 26, 693 (1955).

7. G. K. White, "Experimental Techniques in Low-Temperature Physics", Oxford University Press, London, 1959.

8. J. R. Clement and E.H. Quinnel1, Rev. Sci. Instr., 23, 213 (1952).

9. P. G. Klemens, Proc. Roy. Soc. (London), A208, 108 (1951).

10. J. E. Tucker and W. Reese, J. Chem. Phys., 46, 1388 (1967).

11. A. Keller, Kolloid-Z., 197, 98 (1964); ibid., 231, 386 (1969).

12. H. D. Keith and F. J. Padden, J. Polym. Sci., 39, 101, 123 (1959).

13. R. Hosemann, J. Polym. Sci., Part C, No. 20, 1 (1967).

14. W. O. Statton, J. Polym. Sci., Part C, No. 18, 33 (1967).

15. S. Kavesh and J. M. Schultz, J. Polym. Sci., Part A-2, 8, 243 (1970).

16. M. Matsui and Y. Wada, Rept. Progr. Polym. Phys. Japan, 13, (1970), to be published.

17. P. H. Lindenmeyer, J. Polym. Sci., Part C, No. 15, 109 (1966).

18. M. Giles and C. Terry, Phys. Rev. Letters, 22, 882 (1969).

19. I. A. Viktorov, "Rayleigh and Lamb Waves", Plenum Press, New York, 1967, p 67. 